\title{
Se lo sport entra in dialisi la bioetica dove si piazza?
}

\author{
Mario Timio ${ }^{1,2}$, Francesca Timio ${ }^{1}$ \\ ${ }^{1}$ Dipartimento di Medicina Interna e Nefrologia e Dialisi, Ospedale di Foligno, Foligno (PG) \\ ${ }^{2}$ Direzione Scientifica Centro di Bioetica, Regione Umbria, Perugia
}

\begin{abstract}
Sport and dialysis: a changing bioethics outcome
Despite growing consensus among nephrologists on the importance and benefit of exercise and sport to renal patients, the assessment of physical function or the encouragement of physical activity is not a part of the routine therapy program of patients with chronic kidney disease. Lack of motivation and scanty awareness of the beneficial effects of exercise and sport lead nephrologists to underestimate the impact of physical training on improving the quality of life of renal patients. In addition, exercise and sport have a parallel in adequate commitment of medical staff and reinforcement of bioethics practice. This practice contributes to defining the main elements of a sustained training program: the exercise needs to be interesting and stimulating; information delivery is of crucial importance; exercise is not for everyone and requires individual prescription; and age is not a barrier to exercise in hemodialysis and transplantation. Although the dialysis staff is in an excellent position to encourage and facilitate sustained physical activity, they may not have the skills to provide exercise training and will therefore need exercise professionals. Such a professional can coach and encourage dialysis staff to embrace exercise as a part of patient prescription and increase the confidence of doctors and nurses in physical activity and sport. The three testimonies of renal patients presented by the authors confirm the positive trend in quality of life and the increased adaption to current conditions.
\end{abstract}

Keywords: Bioetics message, CKD, Exercise, Sport

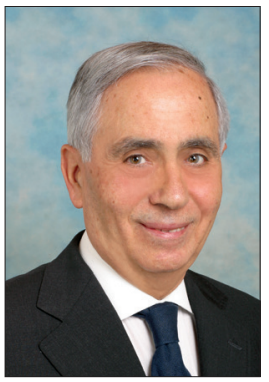

Mario Timio

\section{Trapiantata con la racchetta in mano}

Marta Nizzo (il suo nome è riportato su tutti i giornali locali e nazionali) trentaquattro anni, sposata, amministrativa nella ASL di Perugia (1). Glomerulonefrite, insufficienza renale cronica, emodialisi, trapianto renale dopo tre anni. Sembra un "normale" iter medico che punteggia la storia di tanti nefropatici. Ma non è proprio così. O meglio per Marta manca qualcosa che tanti non posseggono. In positivo. Marta è una tennista che ha riscosso successi nazionali e internazionali. Prima, durante la malattia e dopo il trapianto. Ai World Transplant Games che si sono svolti in Svezia nel 2011 e quest'anno in Spagna (Malaga) nella categoria 30-39

Accepted: July 21, 2017

Published online: September 11, 2017

Indirizzo per la corrispondenza:

Prof. Mario Timio

Via XX Settembre, 22

06100 Perugia

timma@libero.it anni è diventata campionessa del mondo fra i trapiantati. "Lo sport aiuta tantissimo a superare i momenti di difficoltà che trovano le persone che hanno avuto un trapianto - afferma Marta in una intervista rilasciata recentemente a un quotidiano locale - questi eventi internazionali sono importantissimi per sensibilizzare tutti sull'importanza della donazione." Parole molto più efficaci di cento campagne pro-donazione, poiché basate sulla testimonianza diretta così sintetizzata: la malattia, la dialisi, il trapianto possono sostenere iniziative fondate su valori forti e solidali che fanno salire sul podio di qualsiasi sport. Poiché lo sport è vita, è un integratore sociale di primo livello. Lo sport, come la vittoria, "è una sensazione bellissima - prosegue Marta - che fai fatica a realizzare. Ci vuole sempre un po' per renderti conto della bellezza del momento e poi scatta la felicità. Ti paga dei sacrifici fatti e sei orgogliosa di portare la maglia della Nazionale. Ma il pensiero va subito a chi ti ha donato la vita. Gioco a tennis nel suo ricordo e dopo ogni allenamento e ogni vittoria il pensiero corre sempre a colui che mi ha regalato una seconda vita". Dalle parole di Marta emergono messaggi di tipo medico, sociale, bioetico. Sull'effetto benefico dello sport nella prevenzione e cura di alcune patologie come quelle cardiovascolari, la letteratura è esuberantemente ricca. Anche nei nefropatici alcuni esercizi fisici di tipo non-competitivo sono indicati per tenere tonica e trofica la muscolatura, come nelle persone sane (2). Per queste il CSI (Centro Sportivo Ita- 
liano) fa sapere che la promozione sportiva dei suoi iscritti (oltre 450mila) genera un risparmio per il SSN di circa 250 milioni di euro, con proiezione sulla popolazione generale di 2,4 miliardi di euro. Lo sport allora come elemento centrale per la vita delle persone e delle comunità, come fattore di aggregazione interpersonale, di sviluppo relazionale, territoriale, economico, lavorativo. Lo ha realizzato bene Marta e anche I'ANED sport (come segmento della ANED Onlus) che nel gestire la Nazionale trapiantati e dializzati trasmette un messaggio ben preciso di solidarietà e di bioetica, se con questa si intende il prendersi cura della persona-paziente.

Già prendersi cura. Nell'archetipo ippocratico i medici dovevano essere provvisti di philotechnia quanto di philantropia. In altri termini prendersi cura dell'altro significava conoscere la tecnologia medica e la buona pratica per aiutare l'individuo bisognoso. Ove puoi trovare una profonda componente bioetica. Infatti nell'atto del medico ippocratico "I'ispezione del malato coincideva con lo sguardo di simpatia, il tocco della fronte con il contatto fisico, la presa del polso con la stretta della mano, la raccolta dell'anamnesi con l'ascolto del vissuto, la formulazione della prognosi con la risposta alla speranza di vita" (3). Una speranza che oggi può essere attivata consigliando e sostenendo una pratica fisica o addirittura sportiva. Ai nefropatici. Purtroppo la cultura sportiva da suggerire o sostenere tra i nefropatici, siano essi dializzati o trapiantati, non ha avuto una larga diffusione fino a qualche anno fa. È sono allora degni di menzione i risultati di un meeting SIN dal titolo molto esplicativo: Physical Exercise as the first Therapy in CKD (4). II dato più interessante che emerge è il basso livello di esercizio fisico tra i nefropatici. Contestualmente si evidenzia una significativa protezione cardiovascolare nei nefropatici con l'esercizio muscolare. Secondo i dati dello studio DOPPS il rischio di mortalità nei dializzati che espletano attività fisica almeno un volta alla settimana è la metà rispetto ai sedentari (5). II progetto "Transplant... and now it's time for Sport" (6) è uno studio policentrico che ha arruolato 120 trapiantati e ha confermato il ruolo migliorativo della performance fisica e dei parametri della Health-Related Quality of Life (HRQoL). Non sappiamo se Marta abbia fatto parte dello studio, né se tra i trapiantati siano emersi campioni di qualche sport. Ma ciò e irrilevante. L'aver polarizzato l'attenzione sul binomio sport e trapiantati è invece la novità nel campo dei nefropatici, nei quali l'attività fisica è stata sempre sottovalutata.

\section{Quando il pallone non va in rete}

Andrea, ventisette anni, tumore al cervelletto, con grande disabilità fisica e di equilibrio fin dalla adolescenza. In emodialisi per poche settimane a causa di una forma intermittente di insufficienza renale acuta. Tra una seduta e l'altra, quando viene a sapere che sono iuventino, ogni nostro colloquio è improntato quasi interamente sul calcio, anche perché anche lui è un supporter della squadra torinese. Mi narra che da piccolo giocava al calcio con buoni risultati. Poi la malattia come una cesoia taglia ogni speranza di legare la sua vita al calcio.
Ma la speranza di fare il gol della sua vita rimane più tenace che mai. Mi dice che era attaccante e di palloni in rete ne ha posti molti. Un giorno mi dona un libretto di sue poesie. Mi colpiscono due composizioni perché inerenti al suo sport preferito, ma abbandonato. Le riporto ad verbatim.

\section{Gita a Coverciano}

In terza superiore/lo e i miei compagni

siamo andati in gita a Coverciano.

Che meraviglia pestare quei campi

dove si sono allenati i grandi campioni

Bettega Zoff Anastasi.

Che onore poi indossare le loro casacche

Vedere le fotografie/di alcune loro partite.

Anch'io sono un campione

Perché sto vincendo la mia partita

Contro la sofferenza.

\section{Un Campione dei giorni nostri}

\section{Francesco Totti è un campione}

Un giovane talento della Roma

È prezioso per la sua squadra.

Egli non è bravissimo con l'italiano

Per questo è bersagliato dalle barzellette.

Ma quando scende in campo si fa onore.

È anche un po' ingenuo a volte

Ma quando ha il pallone tra i piedi

Si eleva una musica nello stadio.

Rimane comunque un ingenuo

Con il tempo forse cambierà

Ma la sua maglia giallorossa sempre sarà.

Lo critico benevolmente perché il nome dei calciatori a Coverciano sono datati e pochi li ricordano. "Non c'è problema - risponde - avrei potuto citare altre celebrità più attuali che hanno calcato il prato verde di Coverciano, purché utili per la rima". Glisso, e concordo con lui su Totti essenzialmente per la sua fedeltà alla Roma, confermata anche recentemente. Mi faccio spiegare, comunque, il motivo per cui lui, iuventino, ha voluto immortalare in una poesia la figura di un giocatore che seppure famoso è sempre della Roma. "Elementare - mi spiega Andrea - Totti indossa la maglia n.10, la stessa che avevo sulle spalle quando giocavo nella squadretta della parrocchia". In ogni caso con lui il calcio serpeggia spesso in sala dialisi e non solo quello che egli assume per controllare l'ipocalcemia. Dopo la sua dimissione il calcio va in quarantena e chi subentra? La barca a vela.

\section{La barca a vela approda in sala dialisi}

Antonio, cinquantaquattro anni, assicuratore, sempre elegante, modi signorili, eloquio forbito e preciso. In emodialisi 
da quattro anni per malattia policistica renale. È un paziente vacanziero; staziona in riva al lago Trasimeno che solca con una splendida barca a vela di ultima generazione. Al termine di ogni dialisi si ferma nel mio studio e argomenta sullo sport da lui praticato da molti anni. Effettivamente mi trova impreparato sia sui termini tecnici del veleggiare sia sulla consistenza specifica di questo sport. Comunque è così insistente che riesce a sollecitare un certo mio interesse, fino ad accettare il suo invito sulla barca a vela. Così dalle acque della emodialisi alle acque del Trasimeno il passo è stato breve, anche se mi trovavo più a mio agio con le soluzioni di reinfusione che con $\mathrm{i}$ flutti sollevati dall'imbarcazione. Per l'assicuratore è un motivo in più per parlarmi della sua malattia e del suo amore per la vela fin da giovane. Ha perfezionato il suo livello sportivo frequentando una scuola di vela per diversamente abili. Mi parla anche delle finalità del progetto della scuola che consiste nell'elevare la "qualità della vita delle persone con qualche disabilità, sia sul piano dell'autonomia fisica e psicologica, sia sul piano dell'interagire con gli altri e con la realtà circostante per acquisire benessere, sicurezza e identità". Nelle parole di Antonio ci sono tutte le motivazioni che spingono anche un nefropatico a praticare sport e veicolarlo in sala dialisi con le parole, l'entusiasmo, la gioia di vivere. Nella sua stessa narrazione si possono estrarre anche note di bioetica. "L'obiettivo essenziale della scuola di barca a vela - riferisce ancora Antonio - non è quello di far diventare i disabili fenomeni della vela, ma aiutarli a superare le difficoltà, a relazionarsi con gli altri e a integrarsi, godendo della navigazione con il gioco-barca". II velista aggiunge che senza il gioco-barca l'accettazione della dialisi sarebbe stata molto più dura a il conviverci molto più difficile. Allora l'esercizio fisico regolare come cofattore di benessere nei dializzati, spalmato su numerose componenti, come viene suggerito da Aucella (7): a) dà maggiore energia e fa sentirsi in forma; b) migliora sia l'umore che la qualità della vita; c) riduce lo stress, favorisce il sonno e la digestione; d) controlla il peso corporeo; e) contribuisce a regolare la pressione arteriosa; f) riduce il colesterolo e cambia il rischio di cardiopatia; g) rafforza le ossa e la muscolatura. Aggiungo una componente bioetica: favorisce l'interrelazione con altri pazienti, migliora i rapporti con il personale infermieristico e con i medici che si prendono cura del nefropatico.

\section{Conclusioni}

C'è ancora una scarsa cultura sportiva in ambito nefrologico tra i pazienti e il personale, sia per carenza motivazionale che per la percezione che l'esercizio non sia legato al miglioramento della qualità di vita. Ma c'è un fattore che sovrasta tutti gli altri: è il nefrologo conscio che l'esercizio fisico (non dico lo sport) sia un valido cofattore terapeutico durante la storia clinica del nefropatico? Abbiamo riportato l'esperienza sportiva di tre pazienti con malattia renale nella fase di trapianto e di emodialisi, per fornire risposte a molte ritrosie o scetticismo sulla validità o opportunità di esercizio fisico in questa categoria di malati. Marta, Andrea e Antonio hanno squarciato un mondo "liquido" in cui sembra che il pianeta esercizio o sport non possa atterrare sull'universo delle nefropatie. Un valido messaggio clinico in controtendenza che fa emergere un altro parallelo indirizzo bioetico: il nefrologo e il personale tecnico sono nell'eccellente posizione di inserire l'attività fisica nel bagaglio terapeutico di ogni nefropatico, con programmi sostenibili e adattati a ciascuno di loro. Anche con l'ausilio di validi e motivati fisioterapisti. Lo studio Excite (Exercise Introduction to Enhance Performance in Dialysis) fornisce risultati incoraggianti in questo settore e aiuta a vincere la serie di ostacoli fisici e psicologici che intralciano la quotidianità dei dializzati e dei trapiantati. La testimonianza di Marta, Andrea e Antonio rafforza il concetto che se lo sport entra in dialisi la bioetica si piazza nella prime posizioni con un numero vincente di gol. Traspare allora evidente il lavoro che ancora c'è da fare in nefrologia (e in tutta la medicina) cioè di sviluppare un "occhio clinico" non più solo per azzeccare in anticipo una diagnosi, ma per capire la particolarità di una persona che costantemente ha bisogno di aggiornare la diagnosi (8). Anche con le eventuali note di sport. D'altra parte, come sottolineava Aristotele: "La scienza misura le cose, ma a ben vedere le cose misurano la scienza".

\section{Disclosures}

Financial support: No financial support was received for this submission. Conflict of interest: The authors have no conflict of interest.

\section{Bibliografia}

1. Fiorucci L. In campo per sostenere le donazioni. Corriere dell'Umbria, 8 Luglio, 2017.

2. Aucella F, Valente GL, Catizone $\mathrm{L}$. The role of physical activity in the CKD setting. Kidney Blood Press Res. 2014;39(2-3):97-106.

3. Cosmacini G. La qualità del tuo medico. Per una filosofia della medicina. Bari: Laterza 1985.

4. Aucella F, Catizone L. (editors) Physical exercise as the first therapy in CKD. Kidney Blood Press Res 2014;39(2-3).

5. Tentori F, Elder SJ, Thumma J, et al. Physical exercise among participants in the Dialysis Outcomes and Practice Pattern Study (DOPPS): correlates and associated outcomes. Nephrol Dial Transplant. 2010;25(9):3050-62.

6. Mosconi G, Cical V, Tonioli M. Physical activity in solid organ transplant recipients: Preliminary results of the Italian project. Kidney Blood Press Res. 2014;39(2-3):220-7.

7. Aucella F, Gesuete A, Battaglia Y. A "nephrological" approach to physical activity. Kidney Blood Press Res. 2014:39(2-3): 189-96.

8. Traversa G. Per una epistemologia della cura. Keiron Cura; 2003;12:32-7. 